\title{
Space-VLBI as seen from Japan
}

\author{
Hisashi Hirabayashi \\ The Institute of Space and Astronautical Science \\ Japan Aerospace Exploration Agency \\ \#-1-1, Yoshinodai, sagamihara,Kanagawa, 229-8510 Japan \\ E-mail: hirax@tbr.t-com.ne.jp
}

\begin{abstract}
Space-VLBI started to be talked about in 1980s as a small test ISAS mission with small existing launcher. The idea evolved into a larger program with new ISAS launcher development, significant collaboration with NASA Jet Propulsion Laboratory, and with world wide collaborations with ground VLBI facilities. Space-VLBI needed good international coordination, and frameworks for its complex operation were established. IACG (Inter-Agency Consultative Group of Space Science) Panel-1 for Radio Astronomy was formed from spaceagencies side, and GVWG (Global VLBI Working Group) was formed from radio astronomy side. And the Japanese VSOP (VLBI Space Observatory Programme) mission formed VISC, VSOG, etc for the mission as were suggested by the guideline. Richard Schilizzi, with his experience and wisdom contributed in many ways in these activities..
\end{abstract}

\section{Early stage of Japanese space-VLBI planning}

Nobeyama Radio Observatory was dedicated for mm-wave radio astronomy and was opened in 1982. Using the $45 \mathrm{~m}$ radio telescope as a very sensitive element for mm-VLBI and targeting to active galactic nuclei was planned. Small VLBI group started to work on this. After confirming $1.3 \mathrm{~cm}$ fringes with the help of Haystack Radio Observatory, coherency checks were carefully done at Nobeyama 45m telescope, and first international 7mm fringes with Nobeyama was obtained in 1986, and the first 3mm international fringes with Nobeyama followed in 1988. We talked about the future plans after Nobeyama, and I suggested space-VLBI as a possibility even though very challenging. As cosmic radio astronomy in Japan was a newcomer, we wanted to do something new and original rather than doing something already started and established elsewhere.

Prof. Minoru Oda, ISAS director at that time and well-known as the father of ISAS X-ray astronomy group, one day called us for the meeting for space-VLBI. The meeting started with his address; "Is it crazy to think of ISAS-based space-VLBI ? ”, ending with his concluding remark, "Well, it was not proved crazy yet. Why not meeting again ?” This free-talking meeting became the space-VLBI working group which later proposed to ISAS for the mission. His optimism and free mind kept the group active, and Prof. Oda used to be so supportive to space-VLBI during his life. Prof. Oda was one of the first generation radio astronomers in Japan, and he tried to design and build solar radio interferometer with Prof. Takakura before beaming himself to cosmic rays and X-ray astronomy.

ISAS launched two probes, Sakigake and Suisei, for Halley's comet in 1985 with the newly developed M-3S-II rocket. These were the first interplanetary missions by ISAS, and therefore ISAS prepared Usuda 64m antenna for deep space communication, Usuda Deep Space Center, which was dedicated in 1984. And it was only $20 \mathrm{~km}$ from Nobeyama. 
To maximize the scientific return by Halley's probes by Giotto(ESA), two VEGAs(IKI), Sakigake and Suisei(ISAS) and ISEE(NASA) with international coordination, four major space agencies formed IACG, Inter-Agency Consultative Group of Space Science in 1986. There was a recognition that spaceVLBI also needs big international coordination, and Panel-1 for Radio Astronomy, which is for spaceVLBI, was formed at IACG meeting in Padua in October 1986. I was an ISAS delegation member, and had the chance of first meeting of Dave Jauncey, Bob Preston, and Richard Schilizzi, who played very important roles for the activity for many years thereafter. One afternoon we four visited Venice and were greeted by great many pigeons at St. Marco square. The IACG delegation members at Padua were invited by the Pope after the meeting, and met in Vatican to report our activity and received his warm and charming address.

\section{TDRSS-OVLBI Experiment}

Space-VLBI test experiment using existing TDRS satellite was proposed by Gerry Levy of JPL, and Japanese team from NRO, ISAS and CRL (Communication Research Laboratory) joined this. First, the experiment started in S-band, and the Usuda 64m antenna was used as a Japanese main element. There were no VLBI equipments at Usuda, and recording terminal and other necessary electric equipments were detached from Nobeyama 45m telescope and attached to Usuda 64m antenna, This was a good start also for later radio astronomy activity at Usuda using 64m antenna. After successful demonstration at S-band in 1986, 2-cm experiments were successful with the use of the $45 \mathrm{~m}$ telescope as Japanese ground element in 1988, with simultaneous observation at S-band. The TDRSS-OVLBI experiment not only proved the reality of space-VLBI but proved the existence of brightness above inverse-Compton limit, This also helped to form an active and beamed group for space-VLBI in Japan, and helped to convince the credibility of space-VLBI.

\section{Start of VSOP with Muses-B}

I moved to ISAS from NRO in December 1988, and within a month informal announcement of the budget starting from April 1989 was given from the government. Because of many new and difficult engineering items for space-VLBI, the approved space-VLBI satellite and the project was named Muses$\mathrm{B}$, where Muses- is an engineering verification satellite(Mu-Series Engineering Satellite System) Of course scientists wanted to do real observations, and so the mission started with two faces; verifying engineering targets first, and then doing scientific observations.

ISAS started development of new rocket M-V in April 1990 to have 2-5 times morelaunching capability.

The need for wide-band data transfer and phase-link tracking station were significantly different from other satellite projects, and forming the new tracking stations network was a big problem. The SpaceVLBI Project of JPL collaborated extensively to realize the space-mission with wider bandwidth and with good tracking stations coverage and with precise orbit determination. Without this group led by Joel Smith with Bob Preston, the original Muses-B project could not be expanded to full VSOP mission.

There also was a difficulty in convincing the need and preparing correlator for the project at ISAS under some ambiguous situation. But ISAS obtained to build a FX correlator with the heritage of Nobeyama FX correlator in time for the HALCA launch, and was transferred and operated in NAOJ in Mitaka. Eight years were necessary for the launcher development and careful design, fabrication, and detailed testing of the satellite.

\section{VSOP International Collaboration}

Because of the very complex nature of the space-VLBI mission, it was understood that international collaboration and organized collaboration was crucial. 


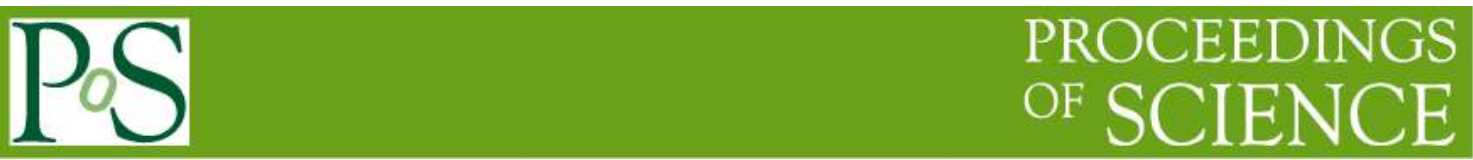

IACG Panel-1 for Radio Astronomy was formed for coming space-VLBI with RadioAstron and VSOP. Frank Jordan and Nikholai Karadashev served long as co-chairs for this. VLBI compatibility issues and mission operation were discussed in detail.

ISAS started as University institute in the beginning, and was running small projects with shorter time scale. But space-VLBI cannot be limited as domestic project. ISAS understood the necessity of forming international science committee, and VISC, VSOP International Science Council, was formed. Already RISC, RadioAstron Science Council, had existed and was a good existing model. Morimoto-san and Toni Zensus served as the first co-chairs, and later, David Jauncey and myself served long until the end of VSOP.

GVWG, Global VLBI Working Group, established in URSI/IAU worked for the ground radio astronomy telescopes and correlators to work effectively in the space-VLBI age. Roy Booth called the first meeting in Onsala Radio Observatory, and played a great leadership for the commitment of radio telescopes for space-VLBI missions. Richard Schilizzi and then Jon Romney succeeded to continue this activity throughout the VSOP mission.

Richard Schilizzi, experienced as QUASAT PI and VLBI astronomer, served in many ways for the international coordination of space-VLBI projects. He served for IACG Panel-1 as a member, for GVWG as a member and a chair, for RISC as member and chair, for VISC as member. He also stayed at NAOJ in Mitaka for several months, and he educated and inspired young team members in Japan.

\section{VSOP. VSOP-2, and after}

Muses-B was successfully launched on February 121997 as a maiden flight for M-V. After 2 weeks, the radio astronomy antenna was successfully deployed. Space-ground baseline fringes were obtained for Usuda and HALCA via Usuda tracking station with VSOP FX correlator. Soon, 3 baseline closure phase was successfully obtained for HALCA-Usuda-Kashima with good quality. At this stage we proposed ISAS to form Next Generation Space-VIBI Working Group, and it was so approved.

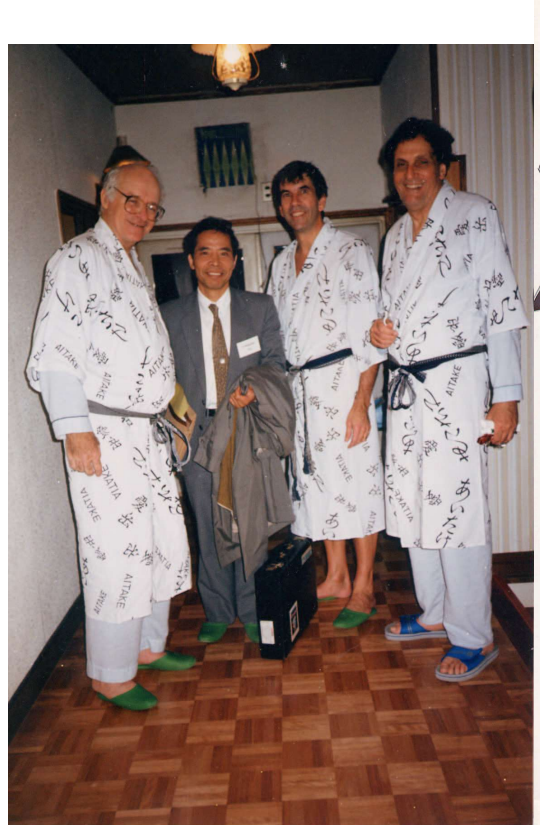

Figure 1. Three wise black ship captains (captains from Woods hall, Holland, and Ventura) (Birnie Burke, Hirax, Richard Schilizzi and Gerry Levy) and a Japanese business man.

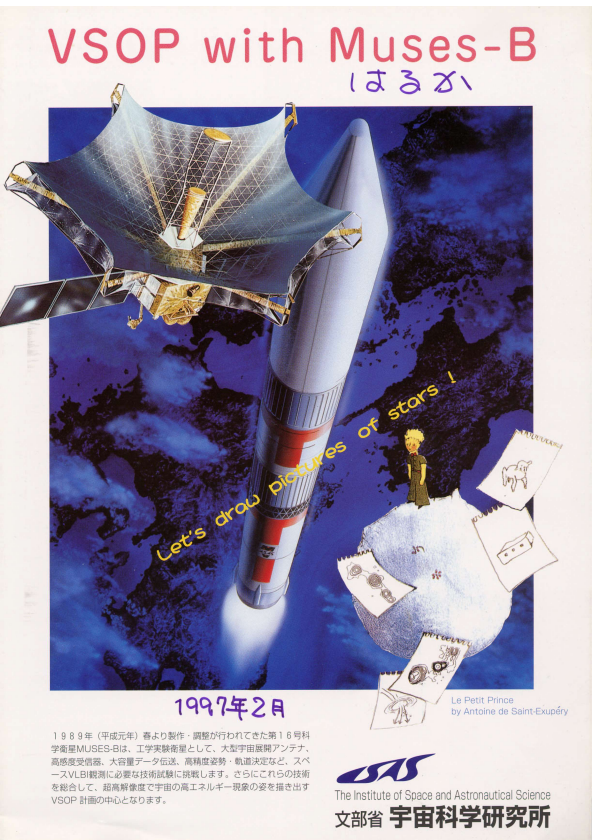

Figure 2. Artist's impression of HALCA and $\mathrm{M}-\mathrm{V}$ rocket 
The first map was generated from HALCA-VLBA experiment in June. And after some more activities, we declared VSOP open in October 1997. VSOP activities and accomplishments are described elsewhere, and so here I just mention that many individual members and institutes collaborated long years for this big endeavor.

VSOP's success gave birth to VSOP-2. HALCA was selected as engineering satellite, yet VSOP was prepared with lots of efforts to work as scientific mission as well. On the other hand, VSOP-2 was proposed and approved as scientific mission. But I saw no major difference between VSOP and VSOP-2. Cancellation of the VSOP-2 is very unfortunate, but ISAS group after the termination of the project in May 2012 is expected to continue future activities.

RadioAstron was launched successfully in 2011 after many years of expectation, and it is very nice to hear that it has started good in-orbit activity. Lessons learned through RadioAstron and VSOP can serve for the future space-VLBI activity. East Asian VLBI Network is being planned and participation for VSOP-2 was in the scope. Chinese space-VLBI is planned, and again, the network could be one of the good ground networks. I hope that we can collaborate globally and Japanese can contribute more.

Reference

Hirabayashi, H. et al, 1998, Science, 281,1825 and erratum 282, 1998

Resolving the Sky - Radio Interferometry: Past, Present and Future

Manchester, UK

April 17-20, 2012 